\title{
NON-COOPERATIVE IRIS RECOGNITION: A NOVEL APPROACH FOR SEGMENTATION AND FAKE IDENTIFICATION
}

\author{
M. Rajeev Kumar, M. Dilsath Fathima, K. Kiruthika and M.S. Saravanan
}

Department of IT, Vel Tech University, Avadi, Chennai, India

Received 2013-07-09; Revised 2013-07-30; Accepted 2013-08-06

\begin{abstract}
Iris recognition, the ability to recognize and distinguish individuals by their pattern, is the most reliable biometric in terms of recognition and identification performance. However, performance of these systems is affected by the heterogeneous images (regarding focus, contrast, or brightness) and with several noise factors (iris obstruction and reflection) when the cooperation is not expectable from the subject. Current Iris recognition system does not deal with the noise data and substantially increase their error rates in these conditions. The non-cooperative iris segmentation takes a vital role in human identification system. This can be simplified with the help of canny edge detection as well as Cartesian to polar conversion methods. An Iris classification method is proposed on the segmented and normalized iris image that divides the image into six regions, followed by independent feature extraction in each region. This will provide the iris signature in terms of binary values, then that are compared with each region for the identification. In addition to this Fake identification is also done in this study. Fake, the original image is forged by fixing lenses over the iris portion.
\end{abstract}

Keywords: Non-Cooperative Iris Recognition, Edge Detection, Cartesian to Polar Conversion, Iris Classification, Feature Extraction, Fake Identification

\section{INTRODUCTION}

The use of biometric systems has been increasingly encouraged by both governments and private entities in order to replace or increase traditional security systems. Biometric is based on a physiological or behavioral characteristic of the person.

A biometric system provides automatic recognition of an individual based on some sort of unique feature or characteristic possessed by the individual. Biometric systems have been developed based on fingerprints, facial features, voice, hand geometry, handwriting, the retina and the iris. Biometric systems work by first capturing a sample of the feature, such as recording a digital sound signal for voice recognition, or taking a digital colour image for face recognition. The sample is then transformed using some sort of mathematical Corresponding Author: M. Rajeev Kumar, Department of IT, Vel Tech University, Avadi, Chennai, India function into a biometric template. The biometric template will provide a normalized, efficient and highly discriminating representation of the feature, which can then be objectively compared with other templates in order to determine identity. Most biometric systems allow two modes of operation. An enrolment mode for adding templates to a database and an identification mode, where a template is created for an individual and then a match is searched for in the database of pre-enrolled templates.

Assuming that, in spite of noise, the iris was accurately segmented, we propose a classification strategy more robust to noise factors. We observed that, in most cases, the noisy data is localized in some of the iris subparts. Our method is based on the division of the segmented iris into six regions, followed by the independent feature extraction in each one. Further, 
through the comparison between signatures extracted from correspondent iris regions, we obtain six dissimilarity values that are fused through a classification rule. The hope is that most of the iris regions are noise-free and that accurate recognition can be achieved, even in highly noisy images.

\section{IRIS RECOGNITION}

Iris is commonly recognized as one of the most reliable biometric measures: it has a random morphogenesis and no genetic penetrance. The iris is a protected internal organ of the eye, located behind the cornea and the aqueous humor. It is the only internal organ of the body that is normally visible externally. Images of the iris adequate for personal identification with very high confidence can be acquired from distances of up to about 3 feet (1 meter). The human iris begins to form during the third month of gestation. The structures creating its distinctive pattern are complete by the eighth month of gestation. In fact, the iris patterns are characterized by high level of stability and distinctiveness. Each individual has a unique iris; the difference even exists between identical twins and between the left and right eye of the same person.

The overall iris recognition system can be given by Fig. 1.

Flom and Safir (1987) studied the problem and concluded that iris morphology remains stable through all human life, as well estimated the probability for two similar irises on distinct persons at 1 in 1072 .

The cooperative behavior demanded to the users and the highly constrained imaging conditions (Rankin et al., 2012) clearly restrict the range of domains where iris recognition can be applied. It is highly probable that image capturing on less constrained conditions (either ata-distance, on-the-move, with minor users' cooperation and within dynamic imaging environments) lead to the appearance of extremely heterogeneous images and with several other types of data in the captured iris regions (e.g., iris obstructions due to eyelids or eyelashes, reflections, off-angle or motion blurred images).

The emerging needs for a safer and quicker access (buildings, weapons and restricted areas) requires noncooperative techniques. In this study, we consider a noncooperative technique where the user has no active participation in the image-capture process and is not even aware of the recognition process.

As an example, we can think of a building access where users do not need to look through a small hole to have their irises recognized, but instead, an imagecapture system captures the necessary information from their irises as they approach the door. This is much less invasive and will enable the dissemination of iris recognition systems to everyday applications. Obviously, these image-capture conditions tend to acquire images with more heterogeneous characteristics with respect to reflection areas, brightness and contrast or focus conditions.

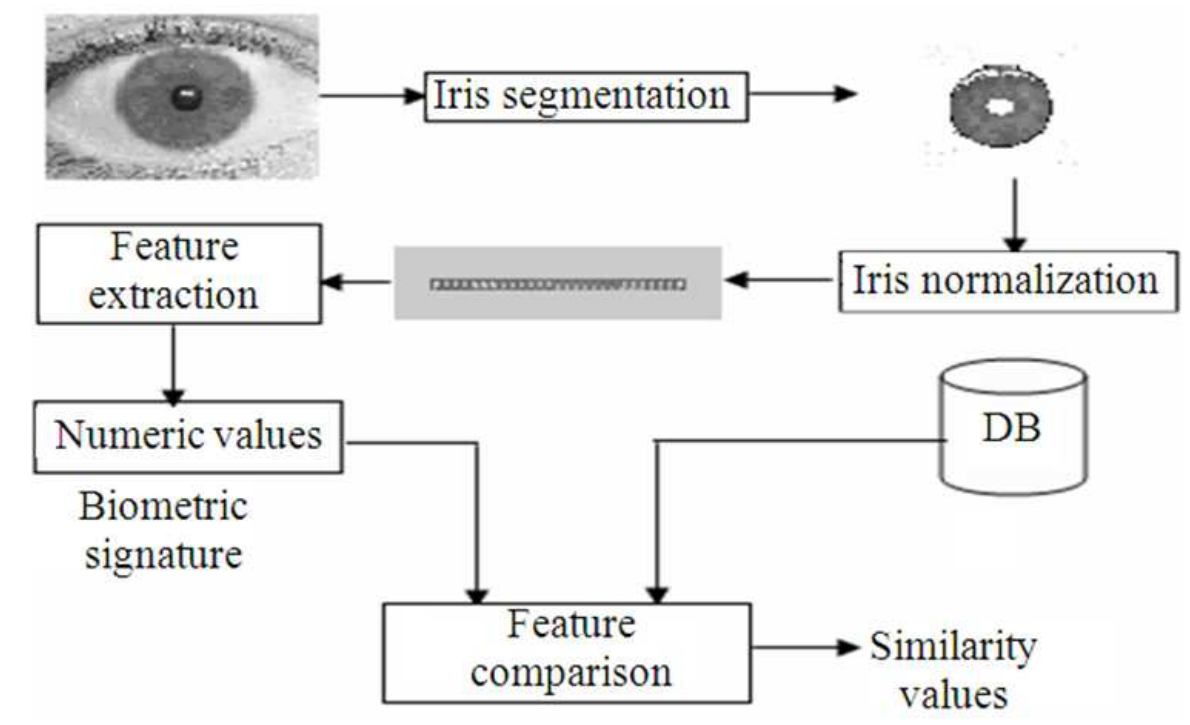

Fig. 1. Stages of iris recognition 
The first stage of the iris recognition is the iris Acquisition. The eye image can be obtained by using CCD Cameras. For the academic purpose, The CASIS, MMU and the UBIRIS (Chinese academic) provided about thousands of iris images in free of cost. We use both of these CASIA, MMU and UBIRIS databases.

After the image acquisition, the next stage of the iris recognition deals with iris segmentation. This consists of localizing the iris inner (pupillary) and outer (scleric) borders. Daugman (1993) proposed an integrodifferential operator to find both the iris inner and outer borders. Similarly, Camus and Wildes (2002) proposed integro-differential operators that search over the IN3 space, with the goal of maximizing the equations that identify the iris borders. Wildes achieved iris segmentation through a gradient-based binary edge map (Sazonova et al., 2012) construction followed by circular Hough transform. Proenca and Alexandre (2006; 2007) proposed a method based on Wildes' method, which, together with a clustering process, achieved robustness for noncooperative environments.

It is possible to varying the pupil's size depending upon various images and in the imaging distance. In order to compensate these variations, it is usual to translate the segmented iris region into a fixed length and dimensionless polar coordinate system. This stage is usually accomplished through the method proposed by Daugman. The method is termed as Daugman's Rubber Sheet Model.

From the view point of feature extraction, previous iris recognition method can be roughly divided into three major characteristics: Phase-based method, Zerocrossing representation method and texture-analysis based method. Daugman used multiscale quadrature wavelet to extract texture phase structure information of the iris to generate a 2,048-bit iriscode and compared the difference between a pair of iris representation by computing their Hamming distance.

Finally, the obtained each signature belongs to different irises. Otherwise, the system outputs a match, meaning that both signatures were extracted from the same iris. In this stage, it is common iris signature is compared with the database, producing a numeric dissimilarity values. If this value is higher than a threshold, the system outputs a non-match, meaning that to apply different distance metrics (Hamming, Euclidean, Weighted Euclidean), or methods based on signal correlation.

\section{RELATED WORKS}

\subsection{Iris Segmentation}

\subsubsection{Daugman Integro-Differential Operator}

Daugman $(2004 ; 1993)$, makes use of an Integrodifferential operator for locating the circular iris and pupil regions and also the arcs of the upper and lower eyelids. The Integro-differential operator is defined as:

$$
\max _{\mathrm{r}, \mathrm{x}_{0}, \mathrm{y}_{0}}\left|\mathrm{G}_{\sigma}(\mathrm{r}) * \frac{\delta}{\delta \mathrm{r}} \int_{\mathrm{r}, \mathrm{x}_{0} \mathrm{y}} \frac{\mathrm{I}(\mathrm{x}, \mathrm{y})}{2 \pi \mathrm{r}} \mathrm{ds}\right|
$$

where, $\mathrm{I}(\mathrm{x}, \mathrm{y})$ is the eye image, $\mathrm{r}$ is the radius to search for, $G_{\sigma}(r)$ is a Gaussian smoothing function and $s$ is the contour of the circle given by $\mathrm{r}, \mathrm{x}_{0}, \mathrm{y}_{0}$. The operator searches for the circular path where there is maximum change in pixel values, by varying the radius and centre $\mathrm{x}$ and $\mathrm{y}$ position of the circular contour. The operator is applied iteratively with the amount of smoothing progressively reduced in order to attain precise localization. Eyelids are localized in a similar manner, with the path of contour integration changed from circular to an arc.

\subsection{Camus and Wildes' Method}

This algorithm (Camus and Wildes, 2002) can be used to find the subject's iris in a close-up image. This algorithm searches in an $\mathrm{N}^{3}$ space for the circumference parameters (centre $(\mathrm{x}, \mathrm{y})$ and radius $\mathrm{z}$ ) by the following function:

$$
\mathrm{C}=\sum_{\theta=1}^{\mathrm{n}}\left((\mathrm{n}-1)\left\|\mathrm{g}_{\theta, \mathrm{r}}\right\|-\sum_{\phi=\theta+1}^{\mathrm{n}}\left\|\mathrm{g}_{\theta, \mathrm{r}}-\mathrm{g}_{\phi, \mathrm{r}}\right\|-\frac{\mathrm{I}_{\theta, \mathrm{r}}}{\mathrm{n}}\right)
$$

where, $\mathrm{n}$ is the total number of directions and $\mathrm{I}_{\theta, \mathrm{r}}$ and $\mathrm{g}_{\theta, \mathrm{r}}$ are, respectively, the image intensity and derivatives with respect to the radius in the polar coordinate system. This methodology is very accurate on images where the pupil and iris regions intensities are clearly separated from the sclera ones and on images that contain no reflections or other noise factors. When dealing with noisy data, the algorithm's accuracy deteriorates significantly.

\subsection{Tuceryan's Methodology}

Tuceryan (1994) proposed a moment-based texture segmentation algorithm, using the moments in small windows of the image as texture features and then applying a clustering algorithm to segment the image. 
The second-order regular geometric moments for each pixel in the image are computed using:

$$
\mathrm{M}_{\mathrm{pq}}=\sum_{-\mathrm{W} / 2}^{\mathrm{W} / 2}\left(\sum_{-\mathrm{W} / 2}^{\mathrm{W} / 2}\left(\mathrm{I}(\mathrm{m}, \mathrm{n}) \mathrm{x}_{\mathrm{m}}^{\mathrm{p}} \mathrm{y}_{\mathrm{n}}^{\mathrm{q}}\right)\right)
$$

where, $\mathrm{M}_{\mathrm{pq}}$ is the regular geometric moment of order $\mathrm{p}_{\mathrm{q}}$, $\mathrm{I}(\mathrm{m}, \mathrm{n})$ the pixel image intensity, $\mathrm{x}, \mathrm{y}$ the neighborhood window coordinates and $\mathrm{W}$ the width. Tuceryan (1994) concluded that these regular moments did not have sufficient discriminant capacity and proposed the application of the hyperbolic tangent as a non-linear transducer followed by an averaging step:

$$
\mathrm{F}_{\mathrm{pq}}(\mathrm{i}, \mathrm{j})=\frac{1}{\mathrm{~L}^{2}} \sum_{(\mathrm{a}, \mathrm{b}) \in \mathrm{w}_{\mathrm{ij}}}\left(\tanh \left(\sigma\left(\mathrm{M}_{\mathrm{pq}}(\mathrm{a}, \mathrm{b})-\overline{\mathrm{M}}\right)\right)\right)
$$

where, $F_{p q}$ is the feature image of the $\mathrm{M}_{\mathrm{pq}}$ moments with mean $\mathrm{M}$ and $\mathrm{W}_{\mathrm{ij}}$ is an $\mathrm{L} \times \mathrm{L}$ average window centered at location (i, j). $\sigma$ is a parameter that controls the shape of the logistic function and was determined, by trial and error, as 0.01 for most cases. At the classification stage, the well-known clustering $\mathrm{K}$-means algorithm is applied producing as output the $n$ clusters-labeled image.

\subsection{Normalization}

\subsubsection{Daugman's Rubber Sheet Model}

The homogenous rubber sheet model devised by Daugman $(2004 ; 1993)$ remaps each point within the iris region to a pair of polar coordinates $(r, \theta)$ where $r$ is on the interval $[0,1]$ and $\theta$ is angle $[0,2 \pi]$. The remapping of the iris region from $(\mathrm{x}, \mathrm{y})$ Cartesian coordinates to the normalized non-concentric polar representation is modeled as:

$$
\mathrm{I}(\mathrm{r}, \theta)=\mathrm{I}(\mathrm{X}(\mathrm{r}, \theta), \mathrm{Y}(\mathrm{r}, \theta))
$$

With:

$$
\begin{aligned}
& X(r, \theta)=(1-r) X_{p}(\theta) r X_{p}(\theta)+r X_{i} \theta \\
& Y(r, \theta)=(1-r) Y_{p}(\theta)+r Y_{i}(\theta)
\end{aligned}
$$

where, $\mathrm{I}(\mathrm{x}, \mathrm{y})$ is the iris region image, $(\mathrm{x}, \mathrm{y})$ are the original Cartesian coordinates, $(r, \theta)$ are the corresponding normalized polar coordinates and are the coordinates of the pupil and iris boundaries along the $\theta$ direction. The rubber sheet model takes into account pupil dilation and size inconsistencies in order to produce a normalized representation with constant dimensions. In this way the iris region is modeled as a flexible rubber sheet anchored at the iris boundary with the pupil centre as the reference point. This is represented in Fig. 2.

\subsection{Image Registration}

The system employs an image registration technique, which geometrically warps a newly acquired image, into alignment with a selected database image. When choosing a mapping function to transform the original coordinates, the image intensity values of the new image are made to be close to those of corresponding points in the reference image. Even though the homogenous rubber sheet model accounts for pupil dilation, imaging distance and non-concentric pupil displacement, it does not compensate for rotational inconsistencies. In the Daugmann system, rotation is accounted for during matching by shifting the iris templates in the $\theta$ direction until two iris templates are aligned.

The mapping function must be chosen so as to minimize:

$$
\int_{x} \int_{y}\left(I_{d}(x, y)-I_{a}(x-u, y-v)\right)^{2} d x d y
$$

While being constrained to capture a similarity transformation of image coordinates $(\mathrm{x}, \mathrm{y})$ to $\left(\mathrm{x}^{\prime}, \mathrm{y}^{\prime}\right)$, that is:

$$
\left(\begin{array}{l}
x^{\prime} \\
y^{\prime}
\end{array}\right)=\left(\begin{array}{l}
x \\
y
\end{array}\right)-\operatorname{sR}(\phi)\left(\begin{array}{l}
x \\
y
\end{array}\right)
$$

With s a scaling factor and $\mathrm{R}(\varphi)$ a matrix representing rotation by $\varphi$. In implementation, given a pair of iris images $I_{a}$ and $I_{d}$ the warping parameters $s$ and $\varphi$ are recovered via an iterative minimization procedure.

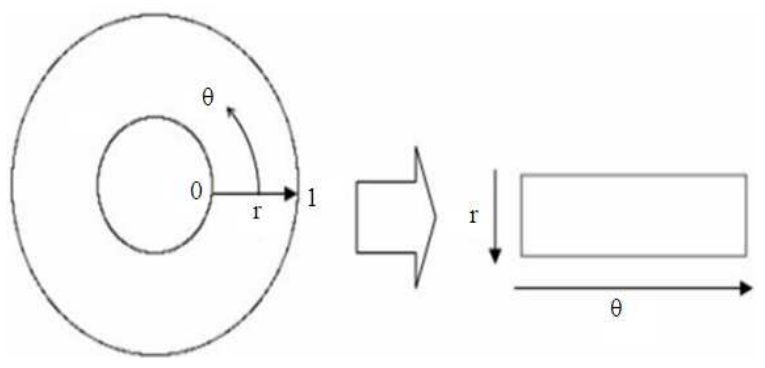

Fig. 2. Rubber sheet model 


\subsection{Iris Coding}

\subsubsection{Gabor Filters}

Gabor filters (Gabor, 1946) are able to provide optimum conjoint representation of a signal in space and spatial frequency. A Gabor filter (Wang et al., 2012) is constructed by modulating a sine/cosine wave with a Gaussian. This is able to provide the optimum conjoint localization in both space and frequency, since a sine wave is perfectly localized in frequency, but not localized in space. Modulation of the sine with a Gaussian provides localization in space, though with loss of localization in frequency. Decomposition of a signal is accomplished using a quadrature pair of Gabor filters, with a real part specified by a cosine modulated by a Gaussian and an imaginary part specified by a sine modulated by a Gaussian.

The real and imaginary filters are also known as the even symmetric and odd symmetric components respectively. The centre frequency of the filter is specified by the frequency of the sine/cosine wave and the bandwidth of the filter is specified by the width of the Gaussian. Daugmann makes uses of a 2D version of Gabor filters in order to encode iris pattern data. A 2D Gabor filter over the an image domain $(\mathrm{x}, \mathrm{y})$ is represented as:

$$
\mathrm{G}(\mathrm{x}, \mathrm{y})=\mathrm{e}^{-\prod\left[\left(\mathrm{x}-\mathrm{x}_{0}\right)^{2} / \alpha^{2}+\left(\mathrm{y}-\mathrm{y}_{0}\right)^{2} / \beta^{2}\right]} \mathrm{e}^{-2 \prod \mathrm{i}\left[\mathrm{u}_{0}\left(\mathrm{x}-\mathrm{x}_{0}\right)+\mathrm{v}_{0}\left(\mathrm{y}-\mathrm{y}_{0}\right)\right]}
$$

where, $\left(\mathrm{x}_{0}, \mathrm{y}_{0}\right)$ specify position in the image, $(\alpha, \beta)$ specify the effective width and length and $\left(\mathrm{u}_{0}, \mathrm{v}_{0}\right)$ specify modulation, which has spatial frequency $\omega_{0}=2020 \mathrm{vu}+$. The odd symmetric and even symmetric 2D Gabor filters are shown in below Fig. 3.

Daugmann demodulates the output of the Gabor filters in order to compress the data. This is done by quantizing the phase information into four levels, for each possible quadrant in the complex plane. It has been shown by Oppenheim and Lim that phase information, rather than amplitude information provides the most significant information within an image. Taking only the phase will allow encoding of discriminating information in the iris, while discarding redundant information such as illumination, which is represented by the amplitude component.

These four levels are represented using two bits of data, so each pixel in the normalized iris pattern corresponds to two bits of data in the iris template. A total of 2,048 bits are calculated for the template and an equal number of masking bits are generated in order to mask out corrupted regions within the iris. This creates a compact 256-byte template, which allows for efficient storage and comparison of irises. The Daugmann system makes use of polar coordinates for normalization, therefore in polar form the filters are given as:

$$
\mathrm{H}(\mathrm{r}, \theta)=\mathrm{e}^{-\mathrm{i} \omega\left(\theta-\theta_{0}\right)} \mathrm{e}^{-\left(\mathrm{r}=\mathrm{r}_{0}\right)^{2} / \alpha^{2}} \mathrm{e}^{-\mathrm{i}\left(\theta-\theta_{0}\right)^{2} / \beta^{2}}
$$

where, $(\alpha, \beta)$ are the same as in above and $\left(\mathrm{r}_{0}, \theta_{0}\right)$ specify the centre frequency of the filter.

\subsection{Log-Gabor Filters}

A disadvantage of the Gabor filter (Gabor, 1946) is that the even symmetric filter will have a DC component whenever the bandwidth is larger than one octave. However, zero DC components can be obtained for any bandwidth by using a Gabor filter which is Gaussian on a logarithmic scale; this is known as the Log-Gabor filter. The frequency response of a Log-Gabor filter is given as:

$$
G(f)=\exp \left(\frac{-\left(\log \left(f / f_{0}\right)\right)^{2}}{2\left(\log \left(\sigma / f_{0}\right)\right)^{2}}\right)
$$

where, $\mathrm{f}_{0}$ represents the centre frequency and $\sigma$ gives the bandwidth of the filter.

\subsection{Zero-Crossings of the 1D Wavelet}

1D wavelets are used for encoding iris pattern data. The mother wavelet is defined as the second derivative of a smoothing function $\theta(\mathrm{x})$ :

$$
\psi(x)=\frac{d^{2} \theta(x)}{d x^{2}}
$$

The zero crossings of dyadic scales of these filters are then used to encode features. The wavelet transform of a signal $f(x)$ at scale $s$ and position $x$ is given by:

$$
\begin{gathered}
W_{s} f(x)=f *\left(s^{2} \frac{d^{2} \theta(x)}{d x^{2}}\right)(x) \\
=s^{2} \frac{d^{2}}{d x^{2}}\left(f * \theta_{s}\right)(x) \\
\theta_{s}=(1 / s) \theta(x / s)
\end{gathered}
$$




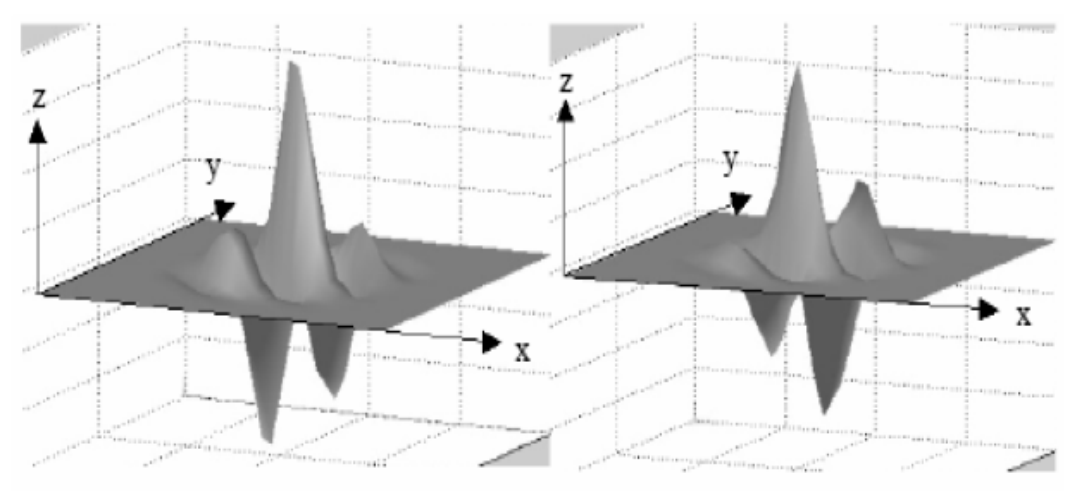

Fig. 3. A quadrature pair of $2 \mathrm{D}$ Gabor filters (left) real component or even symmetric filter characterised by a cosine modulated by a Gaussian (right) imaginary component or odd symmetric filter characterised by a sine modulated by a Gaussian

$\mathrm{W}_{\mathrm{s}} \mathrm{f}(\mathrm{x})$ is proportional to the second derivative of $f(x)$ smoothed by $\theta_{s}(x)$ and the zero crossings of the transform correspond to points of inflection in $\mathrm{f}^{*} \theta_{\mathrm{s}}(\mathrm{x})$. The motivation for this technique is that zero-crossings correspond to significant features with the iris region.

\section{PROPOSED METHODOLOGY}

\subsection{Noncooperative Iris Recognition}

The non-cooperative iris recognition is the process of automatic recognition where individuals using images of their iris captured at a distance and without requiring any active participation. It is shown in the following Fig. 4.

The main drawback of real time implementation of Iris recognition (Burge and Bowyer, 2013) is lies in the segmented image. Where most cases the eye image contains noise such that eyelids, eyelash. These noisy patterns spread across iris and gives less active components of iris patterns. So that the segmentation part of iris is not possibly acquired and it not at all useful for further stages of iris recognition system. The non-cooperative system is possible (Neurotechnology, 2013) by applying a new algorithm namely, multiple signature. i.e., the human iris is going to be divided into six regions.

In the context of non-cooperative recognition (Proenca and Alexandre, 2006; 2007), the most relevant value is the accuracy degradation as function of the images' quality. We observed that our method is clearly less dependent of the image characteristics, since it presented the smallest accuracy degradation between both sessions-just about $0.14 \%$. This is in contrast with all the remaining methods, especially those proposed by Daugman and Camus and Wildes. It must be stressed that our method is the one that presented the highest accuracy on images from the second session, indicating that it is well adapted to deal with noisy images. The multiple signature algorithm based on segmentation is shown in Fig. 5.

In Fig. 5, (a) Division of the iris in four different parts. (b) Division of the iris in "outer" and "inner" parts. (c) Correspondent regions of (a) in the normalized iris image. (d) Correspondent regions of (b) in the normalized iris image.

Our proposal's computation time is about $17 \%$ higher than that of Wildes algorithm; these $17 \%$ are used in the feature extraction and clustering process. We consider that with proper algorithm optimization this computation time gap about 0.3 seconds) will become irrelevant.

Division of the whole iris into six regions is the main concept behind multiple signatures. Here Regions 1 to 4 correspond to successive quadrants of the iris. Regions 5 and 6 correspond, respectively, to the outer and inner parts of the iris.

The main motivation for this division was the observation that the most common types of noise (iris obstructions and reflections) are usual, respectively, in the upper/lower and left/right portions of the iris. Also, reflections resultants from natural and artificial lighting environments are predominantly localized, respectively, in the outer and inner iris regions. The proposed division strategy minimizes the number of regions simultaneously affected by each type of noise. 


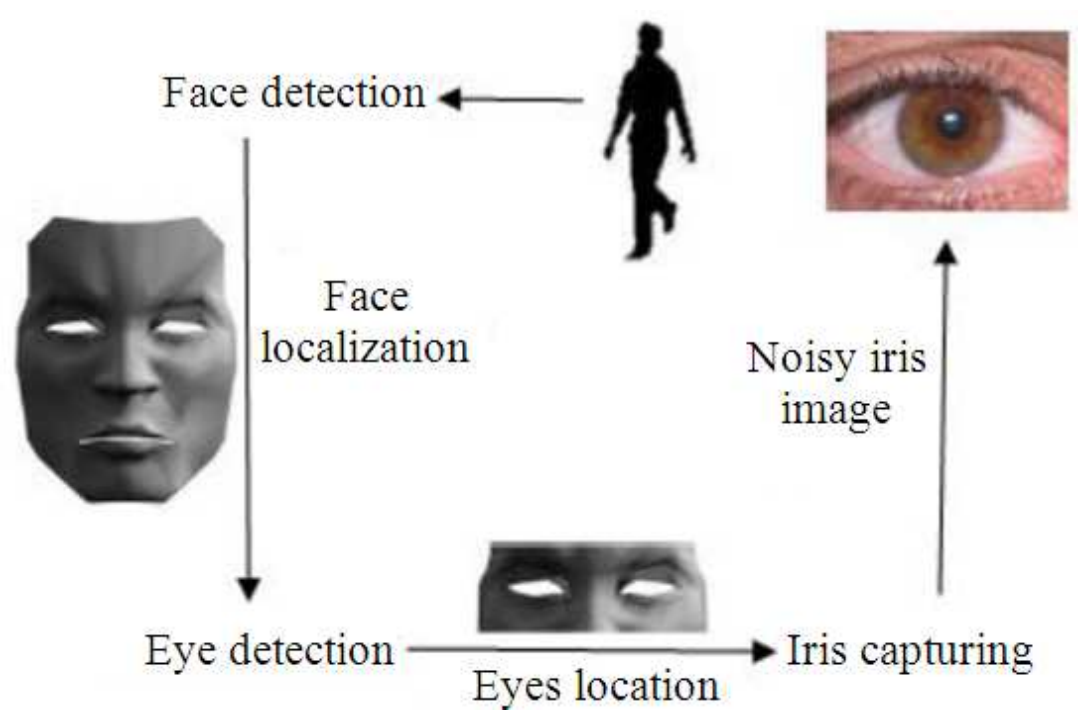

Fig. 4. Non-cooperative system

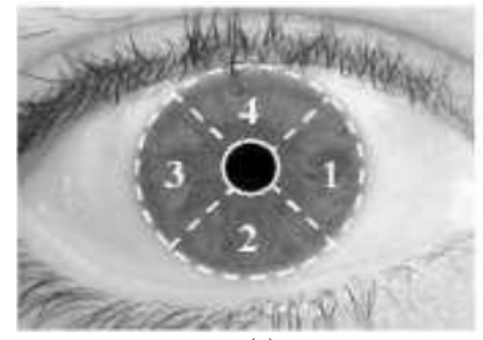

(a)

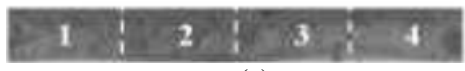

(c)

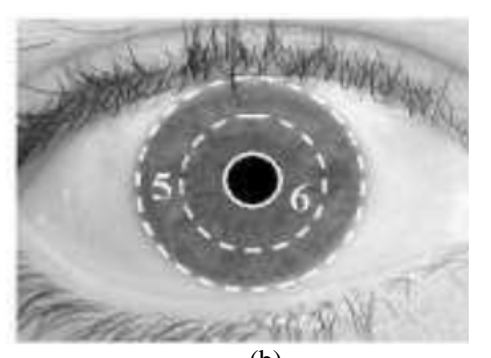

(b)

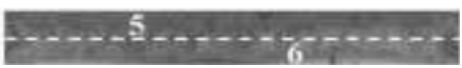

(d)

Fig. 5. Multiple signatures

Based on this, we proposed a new iris classification strategy that divides the segmented and normalized iris into six regions and makes an independent feature extraction and comparison for each of these regions. Iris classification is achieved through a fusion rule that uses a threshold set to combine the dissimilarity values resultant from the comparison between correspondent iris regions.

This indicates that the proposed method is adequate for less constrained image capturing environments, such as in a non-cooperative setting and broadens the range of domains where iris recognition can be applied. However, we stress that these results are dependent on the previous accurate iris segmentation, which is highly challenging, given the dynamics of non-cooperative environments.
The requirement of optical frameworks that are able to capture iris images with enough quality and of real-time face and eye localization methods is assumed too.

\section{IMPLEMENTATION RESULT}

\subsection{Iris Databases}

There are presently seven public and freely available iris image databases for biometric purposes: CASIA, MMU, BATH, UPOL, ICE and UBIRIS (Proenca and Alexandre, 2006; 2007; Phillips et al., 2010; UBIID, 2004). The CASIA database is by far the most widely used for iris biometric purposes. However, its images incorporate few types of noise, almost exclusively related with eyelid and eyelash obstruction, similarly to 
the images from MMU and BATH databases. UPOL images were captured with an optometric framework, obtaining optimal images with extremely similar characteristics. Although ICE and WVU databases contain images with more noise factors, their lack of images (Fenker and Bowyer, 2012) with significant reflections within the iris rings constitutes a weak point regarding the simulation of Non-cooperative imaging conditions. Oppositely, images of the UBIRIS database were captured under natural lighting and heterogeneous imaging conditions, which explains their higher heterogeneity. Based on the manual verification of the iris segmentation accuracy in each of the images, we selected 800 images from 80 subjects of the UBIRIS database.

\subsection{Description of Experiments}

We implemented the recognition method described by Daugman $(2004 ; 1993)$ and compared the obtained results when following the method as described by the author and using the proposed iris division and classification strategies. Initially, we made the feature extraction and comparison using the whole segmented iris, extracting a total of 2,048 bits. Further, according to Fig. 3, we divided the iris into six regions and, through feature extraction, obtained 512 and 1,024 bits, respectively, for the signatures extracted from the iris regions 1 to 4 and 5 to 6 .

The Iris recognition method is divided into the following stages.

\subsection{Segmentation}

The segmentation is the first phase of the Iris recognition. This phase can extract only the iris part from the human eye. We implement the circular edge detection method by using canny edge detector followed by the Cartesian to polar conversion for segmentation.

Initially the segmented result is obtained by removing the pupil and the eyelash. These are removed by using the threshold values as shown in Fig. 6, so the performance of the segmentation is not satisfied. Then the segmentation is done without removing eyelash and pupil.

\subsection{Canny Edge Detection}

Canny edge detection algorithm is to be considering as one among the optimal solution than other based on the detection, localization and number of responses. This algorithm runs in various steps like smoothing, finding gradients, Non-Maximum suppression, double thresholding and edge tracking by hysteresis. The gradient magnitude can be possible by:

$$
\begin{aligned}
& |G|=\sqrt{G_{x}^{2}+G_{y}^{2}} \\
& |G|=G_{x}\left|+G_{y}\right|
\end{aligned}
$$

The direction of the edges must be determined stored as shown below:

$$
\theta=\arctan \left(\frac{\left|\mathrm{G}_{\mathrm{y}}\right|}{\left|\mathrm{G}_{\mathrm{x}}\right|}\right)
$$

\subsection{Cartesian to Polar Conversion}

The Cartesian to polar conversion can be done by the following equation:

$$
r^{2}=x^{2}+y^{2} \quad r=\sqrt{x^{2}+y^{2}} \quad \theta=\tan ^{-1}\left(\frac{y}{x}\right)
$$

\subsection{Normalization}

After the segmentation of both iris borders, to compensate for the varying size of the pupil and capturing distance, we translated the images into a dimensionless polar coordinate system, according to the process known as the Daugman Rubber Sheet (Daugman, 1993; Ma et al., 2004). Generally the segmentation phase will remove the pupil and other than the iris. In order to reduce the complexity of normalization process the segmentation phase itself extracts the required part as shown in (Fig. 4) from the iris. The output of the normalization is given in Fig. 7 .

\subsection{Multiple Signatures}

When we talk about noncooperation (Proenca and Alexandre, 2006; 2007), the captured iris images are normally with the noisy one. That is, most of the images are with obstruction and reflection. So the introduction of multiple signatures is necessary here. Generally the size of the normalized image is $240 \times 20$. This is going to be divided into six regions as four $60 \times 20$ patterns and two $240 \times 10$ patterns. The concept of multiple signatures is given in Fig. 8.

\subsection{Iris Coding}

This iris data encoding was accomplished through the use of two-dimensional Gabor filter. 


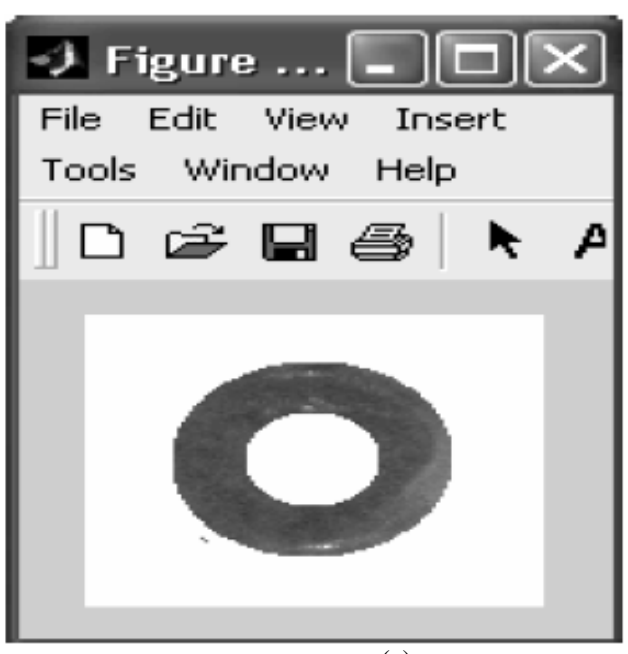

(a)

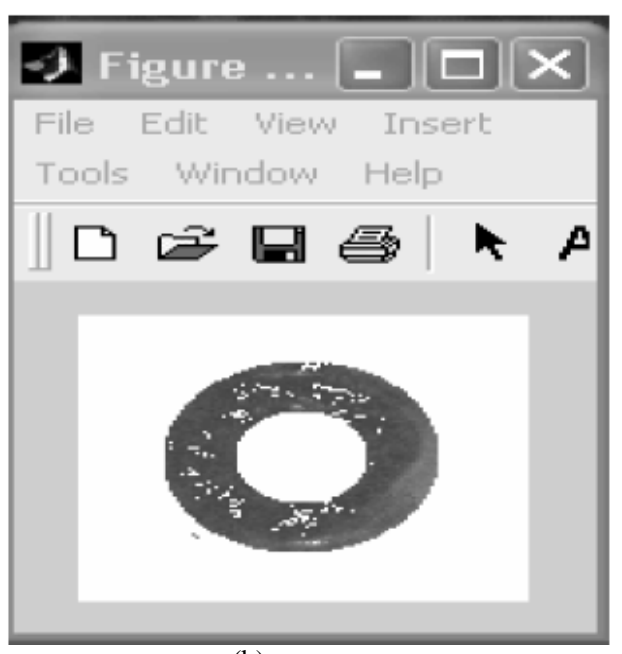

(b)

Fig. 6. (a) Required Segmented Result. (b) Poor Performance Result

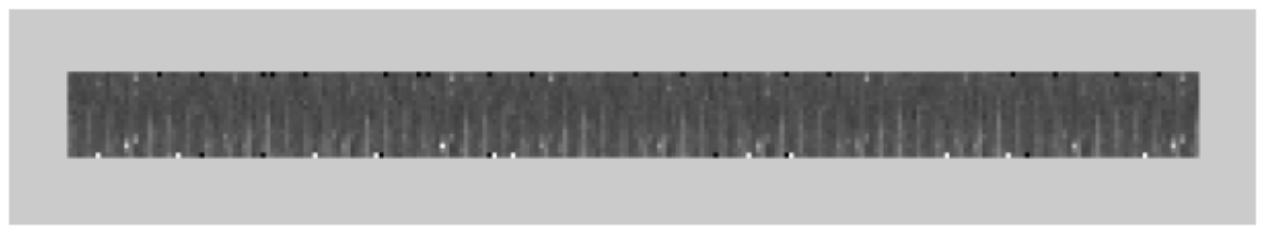

Fig. 7. Required Normalization Result with Size of $240 \times 20$

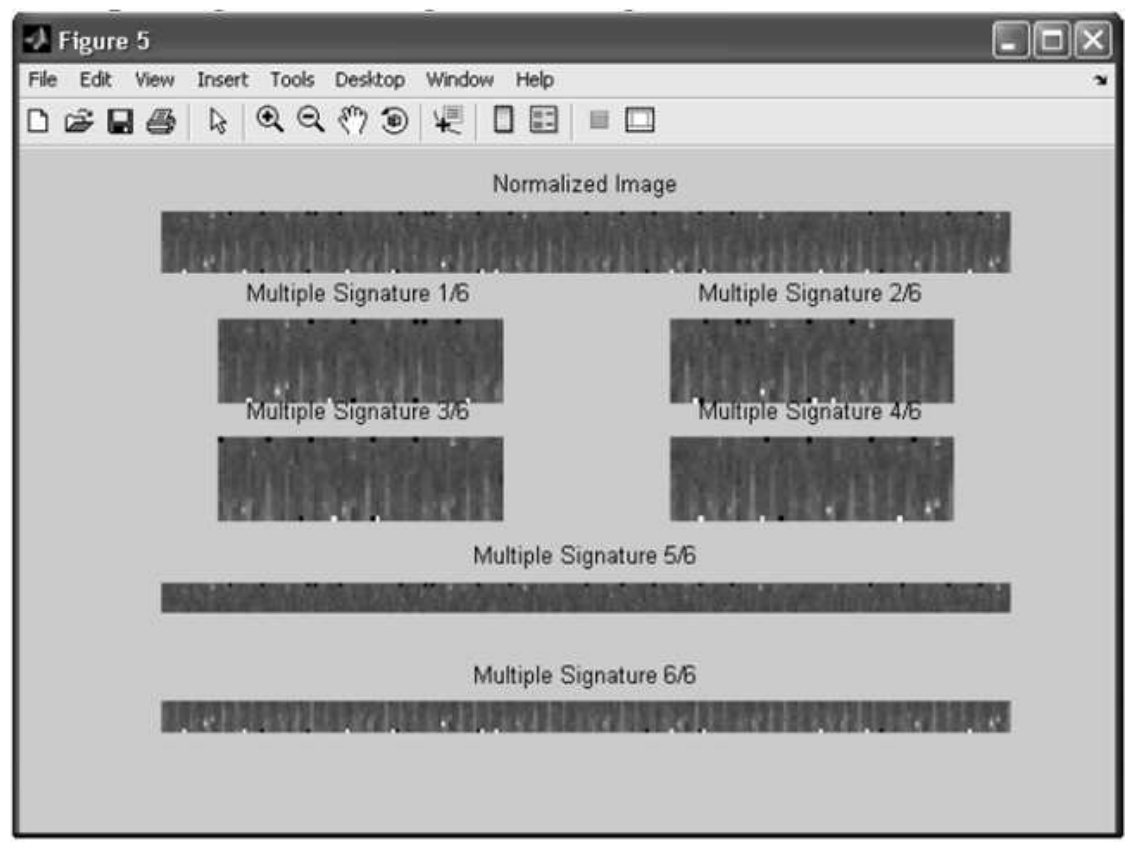

Fig. 8. Multiple Signature $1 / 6,2 / 6,3 / 6,4 / 6$ are $60 \times 20$ patterns and Multiple Signature $5 / 6$ and $6 / 6$ are $240 \times 10$ patterns 


\subsection{Feature Comparison}

The binary feature comparison (Ellavarason and Rathgeb, 2013; Czajka, 2013) allowed the use of the Hamming distance as the similarity measure between two iris signatures. The output of the final iris recognition is given in Fig. 9.

\section{FAKE IDENTIFICATION}

The fake identification module enables the user to find weather the query image is an original or forged one. If the given image is finding to be as a fake one, there is no need for iris recognition for that particular image. This can be identified as given in Fig. 10. That is the difference between the original image and the fake image is shown here. In order to identify the fake image, the Fast Fourier Transform (FFT) is applied on the given image. When the lenses are fixed over the iris portion the quality of the real image is going to be affected. This added advance can be used for fake identification.

The method proposed by Daugman, is probably the most well-known. He proposed the method of using FFT (Kumar et al., 2011) in order to check the high frequency spectral magnitude in the frequency domain, which can be observed distinctly and periodically from the printed iris pattern because of the characteristics of the periodic dot printing. However, the high frequency component cannot be detected in case that input printed iris image is blurred or defocused purposely and the fake iris may be accepted as live one consequently. Therefore, there are two problems concerned, i.e., non-clear (e.g., defocused, motion blurred) and clear printed iris. A system that employs fixed-focus optical lens tends to result in defocused iris images. Motion blurred images are often happens if imitator wobbles purposely when spoofing the iris system.

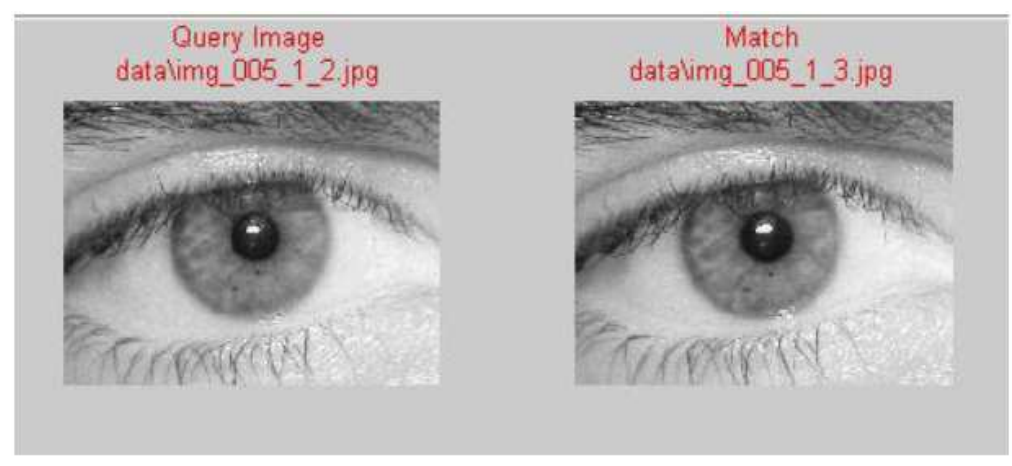

Fig. 9.The input image datalimg_005_1_2.jpg is Matced with the database image datalimg_005_1_3.jpg. Here 005 indicate the 5 th person

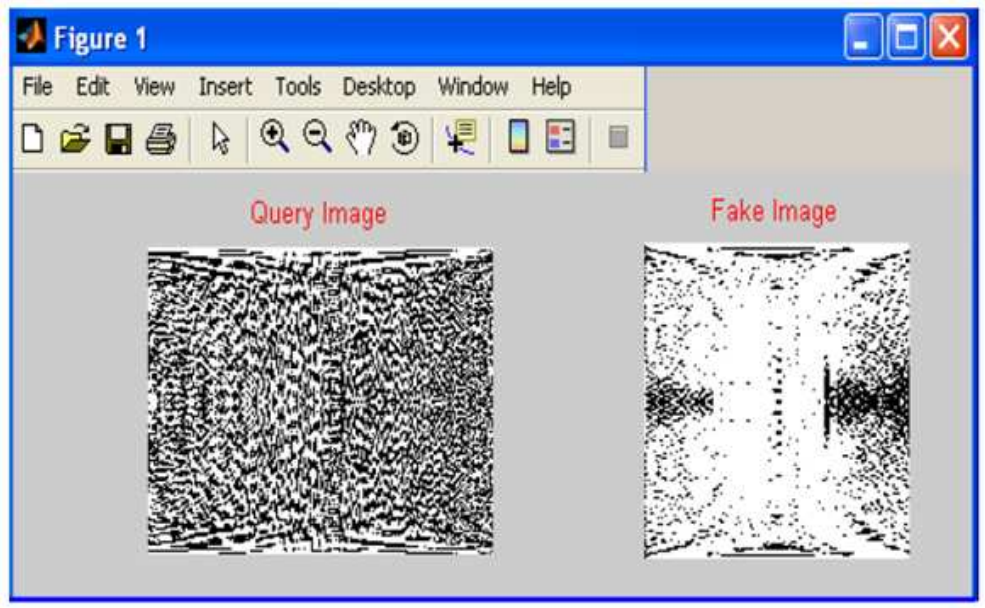

Fig. 10. Comparison of original image with fake image after applying FFT 
Lee et al. (2006) proposed a new method of detecting fake iris attack based on the Purkinje image by using collimated Infra-Red Light Emitting Diode (IR-LED). Especially, they calculated the theoretical positions and distances between the Purkinje images based on the human eye model. However, this method requires additional hardware and need the user's full cooperation.

\section{CONCLUSION}

In this study, we addressed the problems motivated by the existence of noise in the captured iris images and the correspondent increase of the error rates, with particular relevance to the false rejections, in the context of non cooperative iris recognition. The main concentration here is the complexity of the noncooperative iris segmentation. Also fake identification is introduced for the lens images fixing over the iris portion.

\section{REFERENCES}

Burge, M.J. and K.W. Bowyer, 2013. Handbook of Iris Recognition. 1st Edn., Springer-Verlag New York Incorporated, London, ISBN-10: 1447144015, pp: 407.

Camus, T.A. and R. Wildes, 2002. Reliable and fast eye finding in close-up images. Proceedings of the 16th International Conference on Pattern Recognition, (CPR' 02), IEEE Xplore Press, pp: 389-394. DOI: 10.1109/ICPR.2002.1044732

Czajka, A., 2013. Template Ageing in Iris Recognition. Warsaw University of Technology.

Daugman, J., 2004. How iris recognition works. IEEE Trans. Circ. Syst. Video Technol., 14: 21-30. DOI: 10.1109/TCSVT.2003.818350

Daugman, J.G., 1993. High confidence visual recognition of persons by a test of statistical independence. IEEE Trans. Patt. Anal. Mach. Intell., 25: 1148-1161. DOI: 10.1109/34.244676

Ellavarason, E. and C. Rathgeb, 2013. Template ageing in iris biometrics: An investigation of the ND-iristemplate-ageing-2008-2010 database.

Fenker, S.P. and K.W. Bowyer, 2012. Analysis of template aging in iris biometrics. Proceedings of the IEEE Computer Society Conference on Computer Vision and Pattern Recognition Workshops, Jun. 1621, IEEE Xplore Press, Providence, RI, pp: 45-51. DOI: 10.1109/CVPRW.2012.6239214

Flom, L. and A. Safir, 1987. Iris recognition system. US Patent, 4: 394-641.
Gabor, D., 1946. Theory of communication. Part 1: The analysis of information. J. Inst. Elect. Eng., 93: 429441. DOI: $10.1049 /$ ji-3-2.1946.0074

Kumar, M.R., J. Senthil and S. Murugavalli, 2011. Noncooperative iris recognition with fake identification. CiiT Int. J.

Lee, E.C., K.R. Park and J. Kim, 2006. Fake iris detection by using purkinje image. Adv. Biometr., 3832: 397-403. DOI: 10.1007/11608288_53

Ma, L., T. Tan, Y. Wang and D. Zhang, 2004. Local intensity variation analysis for iris recognition. Patt. Recog., 37: 1287-1298. DOI: 10.1016/j.patcog.2004.02.001

Neurotechnology, 2013. VeriEye SDK. Neurotechnology.

Phillips, P.J., W.T. Scruggs, A.J. O'Toole, P.J. Flynn and K.W. Bowyer et al., 2010. FRVT 2006 and ICE 2006 large-scale experimental results. IEEE Trans. Patt. Anal. Mach. Intell., 32: 831-846. DOI: 10.1109/TPAMI.2009.59

Proenca, H. and L.A. Alexandra, 2007. Toward noncooperative iris recognition: A classification approach using multiple signatures. IEEE Trans. Patt. Anal. Mach. Intell., 29: 607-612. DOI: 10.1109/TPAMI.2007.1016

Proenca, H. and L.A. Alexandre, 2006. Iris segmentation methodology for non-cooperative recognition. IEE Proc. Vis. Image Signal Proc., 153: 199-205. DOI: 10.1049/ip-vis:20050213

Rankin, D.K., B.W. Scotney, P.J. Morrow and B.K. Pierscionek, 2012. Iris recognition failure over time: The effects of texture. Patt. Recog., 45: 145-150. DOI: 10.1016/j.patcog.2011.07.019

Sazonova, N., F. Hua, X. Liu, J. Remus and A. Ross et al., 2012. A study on quality-adjusted impact of time lapse on iris recognition. Proceedings of the SPIE International Society for Optical Engineering, (SOE' 12), SPIE, Bellingham, Wash., 83711W-1-83711W9. DOI: $10.1117 / 12.919642$

Tuceryan, M., 1994. Moment-based texture segmentation. Patt. Recog. Lett., 15: 659-668. DOI: 10.1016/0167-8655(94)90069-8

UBIID, 2004. University of Bath Iris Image Database. University of Bath.

Wang, Q., X. Zhang, M. Li, X. Dong and Q. Zhou et al., 2012. Adaboost and multi-orientation 2D Gaborbased noisy iris recognition. Patt. Recog. Lett., 33: 978-983. DOI: 10.1016/j.patrec.2011.08.014 